\title{
Linx
}

Revue des linguistes de l'université Paris X Nanterre

$57 \mid 2007$

Études de syntaxe : français parlé, français hors de

France, créoles

\section{Interrogatives en que en français parlé au Cameroun : approche macro-syntaxique}

\section{Emmanuel Ngué um}

\section{(2) OpenEdition}

\section{Journals}

Édition électronique

URL : http://journals.openedition.org/linx/286

DOI : $10.4000 /$ linx.286

ISSN : 2118-9692

Éditeur

Presses universitaires de Paris Nanterre

Édition imprimée

Date de publication : 1 décembre 2007

Pagination : 113-122

ISSN : 0246-8743

Référence électronique

Emmanuel Ngué um, « Interrogatives en que en français parlé au Cameroun : approche macrosyntaxique », Linx [En ligne], 57 | 2007, mis en ligne le 15 février 2011, consulté le 15 mai 2020. URL: http://journals.openedition.org/linx/286 ; DOI : https://doi.org/10.4000/linx.286 


\title{
Interrogatives en que en français parlé au Cameroun : approche macro-syntaxique
}

\author{
Emmanuel Ngué um \\ Université de Yaoundé 1 et Université de Provence
}

On rencontre souvent, dans les productions orales spontanées de locuteurs du français au Cameroun, des formes interrogatives qui présentent des schémas syntaxiques mettant en relief une organisation particulière de l'énoncé. En voici quelques exemples, dont la transcription est inspirée des conventions du GARS, avec des aménagements notamment pour la marque d'intonation en fin d'énoncé, déterminante dans la reconnaissance des formes interrogatives. Les italiques indiquent les structures dont nous traiterons, et l'ensemble des conventions de transcription figure en fin d'article :

(1) le gars il voit comme ça il fait - on dit que qu'est-ce qu'il est en train de faire là \} (R1.JELA1).

(2) mais on vieillit - tu crois que quoi \qu'on est qu'on est vos vos égaux $\backslash /$ (R5.CYOL)

(3) L1 je suis venu là pour qu'on bagarre $\bigwedge$

L2 ékié ${ }^{\backslash}$ - on bagarre que j'ai la force pour bagarrer $\backslash /$ (R4.CCHRIST)

\footnotetext{
${ }^{1}$ Cette expression est un tour exclamatif emprunté aux langues locales, du groupe Beti-Fang en l'occurrence, exprimant la surprise ou l'exaspération.
} 
Ces constructions affichent deux termes, à savoir une que-structure à valeur interrogative, articulée à un premier terme, segmentalement apparent ou discursivement implicite, que nous désignerons désormais par $\mathrm{P}$.

Nous chercherons à décrire ces structures en précisant la nature du lien syntaxique entre les deux termes, et nous déterminerons aussi le rôle de que dans leur jonction. Nous faisons l'hypothèse que ce lien, non rectionnel, ne peut être décrit en termes morphosyntaxiques. Notre démarche s'inspire de descriptions de l'oral qui prennent en charge aussi bien les indices linguistiques que les indices prosodiques, et se soucient de leur interprétation discursive (Morel \& Danon-Boileau 1998).

Notre analyse s'appuie sur un corpus oral d'environ 6 heures d'enregistrements, correspondant à deux types d'activités : interactions entre amis en contexte informel, et interactions dans une réunion de travail. Pour ce second type, seule la réunion de travail est entièrement transcrite, le reste n'est que partiellement annoté sous PRAAT. Ces enregistrements ont été effectués au Cameroun en 2007 et 2008, dans des conditions écologiques (nous entendons par là que les productions naturelles ne sont aucunement contraintes par la présence du chercheur) pour lesquelles nous avions privilégié des types d'activités interactionnelles plutôt que des profils sociolinguistiques de locuteurs. Nous y ajoutons quelques données recueillies au vol, pour corroborer les observations que l'on peut tirer du corpus.

Après avoir dressé une typologie des que-interrogatives attestées, nous passerons en revue quelques approches rendant compte de ce type de constructions, pour finalement proposer une autre approche.

\section{Typologie des interrogatives en que}

Trois formes de que-interrogatives (désormais que-I) présentant une configuration binaire ont été répertoriées. Une quatrième forme (tu dors que debout $\backslash /$ ) ne sera pas traitée ici. Le premier type correspond à l'exemple (1), auquel nous ajoutons ceux qui suivent :

(4) qu'est-ce que tu voudrais - que le gars t'a qu'on t'abandonne à toi-même comme ça elle me dit alors que / - qui n'a pas encore fait d'enfant $\wedge$ (R1JELA1)

(5) j'ai vu mes camarades tricher à l'enam ${ }^{2}$ - j'étais choquée que comment tu peux tricher à l'enam $\wedge$ (R3. AYALM).

Le deuxième type est représenté par les exemples (2) et (6) :

(6) tu dois croire - les paroles d'un homme - jusqu'à ce que tu aies la preuve: du contraire $\Lambda$ - ça veut dire que quoi $\backslash$ (R1JELA1)

\footnotetext{
${ }^{2}$ Ecole Nationale D’administration et de Magistrature.
} 
Le troisième type enfin correspond aux exemples (3) et (7) :

(7) L3 l'ami de paul est entré dans sa maison samedi passé - une big / $\mathrm{xx}$

$\mathrm{L} 2 \mathrm{hm} \backslash$ - et il est temporaire $\bigwedge$

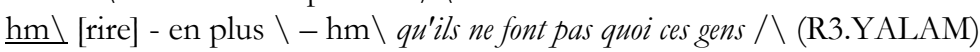

Les que-I sont reconnaissables par les marqueurs qui leur sont attachés (verbaux, intonatifs). Elles constituent des questions par les indices localisables aussi bien au niveau du contenu qu'en relation avec le contexte (contenu propositionnel, savoirs relatifs des participants, autres indices contextuels).

Comme actes de langage, les que-I n'ont pas toujours une force illocutoire de nature à imposer une «obligation juridique " (Ducrot, 1984) à l'interlocuteur; elles n'ont pas non plus pour finalité «d'obtenir [...] un apport d'information» (KerbratOrecchioni, 1991 :14). Si les que-I de type III peuvent être rangées parmi les questions rhétoriques, du fait qu'elles ne préjugent pas d'une réponse, celles de type II oscillent entre vraie question, question rituelle et question rhétorique. Celles du type I en revanche, du fait qu'elles rapportent une question dont la force illocutoire est attribuée à un tiers, n'appellent pas de réponse dans la situation d'énonciation, mais conservent la force illocutoire pour la situation d'énonciation de l'énoncé rapporté.

\subsection{Premier Type $: P+$ que + Pint}

Ces que-I présentent deux termes articulés autour d'une même unité communicative. Les deux termes se présentent sous la forme d'une tournure syndétique, marquée par un que qui pose quelques problèmes d'analyse. Pour certains exemples, il apparait, sur la base d'indices prosodiques, que que n'entre pas dans le même groupe intonatif que le deuxième terme. Dans elle me dit que qui n'a pas encore fait d'enfant en (4), F0 signale une pause entre elle me dit que et qui n'a pas encore fait d'enfant, ce qui montre une articulation intonative non linéaire entre les deux termes. D'autre part, le segment elle me dit que est ponctué par un intonème ouvrant sur que, qui suggère que celui-ci est à rattacher au premier terme, qui constitue ainsi une mise en place pour l'énonciation du deuxième terme.

Toutefois, la binarité de ces constructions ne sera pas fondée sur les seuls critères prosodiques, car l'idée que les profils mélodiques de configurations syntaxiques actualiseraient leur statut micro- au macro- ne fait pas l'unanimité parmi les chercheurs (Avanzi 2007).

Sur le plan énonciatif, les que-I de type I signalent du discours rapporté, ce qui les prédispose sur le plan syntaxique à une configuration de type complétive.

\subsection{Deuxième type: $(P)+$ que + quoi}

Les formes de ce type se situent à mi-chemin entre celles du premier type et celles du troisième type. Elles se rapprochent des premières par le fait qu'elles comportent généralement un verbe recteur de complétive, comme en (2), tu crois que quoi, ou encore vous pensez, que quoi, énoncé relevé au vol. Elles s'en éloignent par le fait qu'elles ne signalent pas du discours rapporté. En outre, les énoncés du deuxième type partagent avec ceux du troisième type une propriété fondamentale: la fréquente 
collocation de que et quoi induisant que quoi. Ils s'en distinguent toutefois par deux critères. Premièrement, le fait que, pour le type II, l'élément que ainsi colloqué à quoi ou à toute autre forme proportionnelle, est toujours introduit par un verbe recteur, même de façon implicite ${ }^{3}$, ce qui n'est pas le cas pour le type III. Deuxièmement, sur le plan pragmatique, les formes que quoi de type II peuvent constituer de véritables questions, ce qui est totalement exclu pour le type III.

\subsection{Le Troisième type $:(P)+$ que + Pint/quoi}

Pour ce type de que-I, il n'est pas possible d'établir un lien rectionnel de quelque nature que ce soit entre la que-structure qui constitue le deuxième terme de la relation binaire, et P. Contrairement aux formes de type II où l'absence d'un verbe recteur peut être simplement imputée à une opération de réduction de $\mathrm{P}$, ici, la questructure jouit d'une parfaite autonomie micro-syntaxique, elle se suffit à elle-même en quelque sorte. Elle reste néanmoins, comme nous le verrons plus loin, discursivement liée à $\mathrm{P}$, dont l'articulation segmentale est soumise aux aléas de la situation du discours et aux besoins communicationnels des locuteurs. C'est ce qui s'observe d'une part en (7), où la que-structure qu'ils ne font pas quoi ces gens $\bigwedge$ enchaîne non sur un terme explicitement articulé, mais sur un savoir partagé inscrit dans la mémoire discursive, et d'autre part en (3) et en (12), où la que-structure s'articule à un premier terme $(\mathrm{P})$ que le locuteur pense devoir rappeler, quoiqu'elle soit tout autant inscrite dans la mémoire discursive. Lorsque que quoi constitue la que-structure d'un énoncé de type III, il ne peut pas être situé sous la rection d'un verbe précédent, même implicitement (cf. (11) vs (12)).

\section{Les que-Phrases dans les français d'Afrique ${ }^{4}$}

Queffélec aborde les que-Phrases dans le discours rapporté par le biais des modalités injonctives et interrogatives dans le français populaire au Cameroun. Rejoignant Italia (2005) pour le français au Gabon, il remarque que «les indices qui permettent à l'énonciateur d'annoncer la présence d'un DR (discours rapporté) et donc le décrochage énonciatif sont atypiques et correspondent à un réaménagement important du français » (2006 : 3). Il affirme, comme l'avaient fait avant lui Ploog (2004) ou Italia (2005), la nature insaisissable de que. Dans l'exemple (8), il soutient que la différence entre discours direct et indirect est rendue caduque par l'absence de transposition de temps verbaux et d'indices de personne, là où, selon lui, « le standard impliquerait : il m'a dit que je (ne) pouvais pas arriver là où nous étions » :

(8) le gars était aussi un gars bandjoun i s'appelait assayo $i$ m'a dit que ma soeur tu peux pas arriver ici que nous sommes (les italiques sont de nous)

\footnotetext{
${ }^{3}$ Comme dans l'exemple (2), où on est vos égaux $\backslash$, proportionnel à quoi, est colloqué à que, et où le verbe recteur n'est recouvrable qu'à travers le premier terme de la structure binaire.

${ }^{4}$ Pour des phénomènes similaires en français hexagonal, voir entre autres Le Goffic, 1992, Deulofeu, 1999, Gadet, 1997.
} 
Ngamountsika (2007) arrive à peu près au même constat sur des exemples de français parlé au Congo, très semblables à nos exemples camerounais, mais qui s'en écartent légèrement, puisqu'ils n'encodent pas la modalité interrogative :

(9) je me suis dit que faites descendre les clients

(10) que moi-même je n'avais dit que non mieux laisser

D’après Ngamountsika (2007 : 174), en (9), «que appuie la sémantèse du verbe régissant. Il n'introduit pas une construction enchâssée. Nous avons donc un discours direct. Que a une valeur de particule énonciative appuyée par le décrocheur énonciatif non».

Souop (2002), sur des données camerounaises, aborde la question sous un angle différent, en recourant aux critères de proportionnalité de la macro-syntaxe pour saisir les relations de dépendance et d'équivalence entre éléments linguistiques :

(11) la Guinness ${ }^{5}$ avait dit que quoi ?

(12) il ne prend pas que quoi ?

S'appuyant sur une analyse distributionnelle et sur le critère de proportionnalité, Souop (2002: 69) détermine que dans certains exemples, quoi est proportionnel à une proforme telle que ça, comme en (11a) :

(11a) la Guinness avait dit ça

La conclusion est ici que, pour ce type d'énoncé, quoi est bien sous la rection du verbe dire en particulier, et en général des verbes recteurs de complétive, d'affirmation, de pensée, de souhait.

Ce rapide parcours suggère l'inadéquation des descriptions antérieures au vu de leurs résultats, ce qui nous conduira à proposer un cadre plus englobant, à partir du modèle macro-syntaxique aixois.

\section{Application du modèle macro-syntaxique}

La première difficulté pour la description des que-I dans le français ordinaire parlé au Cameroun est d'ordre méthodologique. Il faut partir d'une approche modulaire, en faisant la part de ce qui relève de la syntaxe de rection, et de ce qui n'en relève pas. Sinon, on en viendrait à des solutions relevant des niveaux pragmatiques, sémantiques ou discursifs, pour un problème préalablement caractérisé comme morphosyntaxique.

Pour chaque type de que-I recensé, nous nous appuierons sur le modèle macrosyntaxique aixois, notamment en fondant la distinction entre statut micro- ou macrod'une construction sur des critères syntaxiques bien définis. Dans une construction verbale micro-, les éléments sous la rection du verbe ont pour propriétés de pouvoir être : 1) disposés en paradigmes, 2) modulés dans des dispositions syntaxiques, 3) concernés par les modalités du verbe dont ils dépendent.

\footnotetext{
${ }^{5}$ Société brassicole locale.
} 


\subsection{Les formes de type I}

Même si elles ont une configuration "complétivoïde », ces formes réagissent négativement aux tests rectionnels habituels que sont l'extraction, la pronominalisation, et l'application de la modalité négative.

(4a) ? C'est que qui n'a pas encore fait d'enfant qu'elle me dit alors ? elle me dit alors que ça, etc.

? Elle ne me dit pas alors que qui n'a pas encore fait d'enfant

$\mathrm{Si}$, en recherchant la proportionnalité, on fait commuter que qui n'a pas encore fait d'enfant de (4) avec la pro-forme ça, on obtient la construction proportionnelle elle me dit alors ça en (4a), qui est tout à fait acceptable. Mais une telle opération n'est pas possible en (13), où que peut être suivi d'un marqueur discursif (bon), ou même d'une pause :

(13) non mais moi moi ma belle-famille ne peut pas me compliquer $\mathrm{x}$ - ça moi je suis sûr ils vont faire quoi ? - ils ils font ça je leur dis que bon $\backslash$ - restons alors là $\backslash$ (R5.CYOL.)

Il n'est donc pas possible ici d'analyser dire comme un verbe transitif régissant une complétive. Mais il est possible de décrire une proportionnalité entre que et une proforme comme ceci:

(4b) Elle me dit ceci-qui n'a pas encore fait d'enfant

Ceci nous conduit à dire que que est seul sous la rection du verbe, et non l'ensemble de la que-I que qui n'a pas encore fait d'enfant. Il devient alors évident que (4) doit être analysé en une entité macro-syntaxique associant deux éléments, d'une part elle me dit que, et d'autre part qui n'a pas encore fait d'enfant, chacune étant pourvue de ses propres modalités.

Pour la macrosyntaxe aixoise, ces deux termes correspondent à deux noyaux conjoints. Blanche-Benveniste et al (1990) parlent ici de regroupement aléatoire de noyaux. Bien qu'ayant une forme d'autonomie, les noyaux ne peuvent apparaittre isolément sans briser la complétude de l'énoncé. Ainsi, elle me dit que, équivalent de elle me dit ceci, appelle une explication qui est apportée par le deuxième noyau. BlancheBenveniste et al (1990 : 125), observaient que «certains éléments lexicaux, nominaux ou verbaux, ouvrent un rapport d'explication avec un noyau qui les suit». Ce qu'ils exemplifient :

(14) il y a un mystère - par où commencer

(15) on leur pose une question - alors au premier on pose - comme question où et en quelle année a explosé la première bombe atomique

En contexte camerounais, une unité macro-syntaxique peut se réduire au premier noyau, comme dans cet exemple pris au vol, où l'effet suspensif marqué par une intonation ouvrante sur que appelle un second noyau ; mais la charge de l'énonciation est laissée à l'interlocuteur :

dis-moi quel 
Les que-I de type I sont tous construits sur ce paradigme macro-syntaxique, ce qui explique que dans une situation où le discours rapporté est signalé par ce qui parait être un morphème de subordination introduisant le style indirect, nous ayons un discours rapporté littéral, sans transposition des indices temporels et de la personne.

Quand que est ainsi libéré de ses contraintes morphosyntaxiques, il devient possible, sur le module discursif, de lui trouver d'autres fonctionnalités. Dans les exemples que nous venons d'analyser, que annonce une séquence postérieure.

\subsection{Les formes de type II}

Étant donné la régularité de la séquence que quoi en français parlé au Cameroun, il a été proposé (Souop, 2002) de l'analyser comme un morphème unique qui équivaudrait soit à l'adverbe interrogatif pourquoi (elle ne prend pas que quoi en (12)) $)^{6}$, soit à la locution interrogative qu'est-ce que (tu crois que quoi (2)). Que quoi serait alors une forme lexicalisée. S’il y a effectivement une fréquente collocation entre ces deux items, il semble prématuré d'en conclure à la lexicalisation ou au figement. On peut certes argumenter pour un figement, du fait qu'il est impossible d'insérer un élément entre ces deux items (11b) :

$$
\begin{aligned}
\text { (11b) } & \text { - La Guinness avait même dit que quoi } \\
& - \text { La Guinness avait dit que quoi même } \\
& -\quad \text { ? La Guinness avait dit que même quoi }
\end{aligned}
$$

Mais l'association entre que et quoi parait fragile lorsqu'on exploite l'aspect dialogique du discours oral et sa dimension polyphonique, comme en (2a), replacé dans son contexte :

(2a) L1 Michel je l'ai vu avec une touffe de cheveux blancs devant là \ L4 mais ouais non $\backslash /$

L2 mais on vieillit $\bigwedge-$ tu crois que quoi $\backslash$ - qu'on est est qu'on est vos vos égaux $\backslash /$

Une mise en grille montre que ces deux éléments font partie de deux entités discursives différentes, qui, sur le plan interactif, doivent s'associer pour satisfaire à l'exigence de complétude interactive :

\begin{tabular}{|rl|}
\hline tu crois que & quoi $\backslash$ \\
qu' & on est vos égaux $\backslash$ \\
\hline
\end{tabular}

L'argumentation en faveur d'une structure macro-syntaxique reste pourtant délicate, et on peut toujours, pour justifier l'emploi interférent de que, avancer les hypothèses du substrat ou du figement. Mais l'hypothèse substratique ne fournit en elle-même aucune élaboration sur le phénomène en question. D'autre part, s'il y avait figement de que quoi donnant lieu à un morphème équivalent à pourquoi ou à qu'est-ce que, nous aurions du mal à expliquer pourquoi il ne se produit qu'avec certains verbes. Par exemple, nous n'avons pas constaté, même en faisant appel à notre intuition de locuteur du français camerounais, d'énoncés comme tu vois que quoi, au sens des que-I

\footnotetext{
${ }^{6}$ Selon notre classification, l'exemple (12) est à rattacher au que-I de type II.
} 
de type II. Si un tel énoncé devait être attesté, il aurait sans doute un sémantisme différent : il serait à ranger dans les que-I de quatrième zone, que nous avons écartées ici comme n'ayant pas une configuration binaire.

Selon nous, que est dans ces exemples motivé sur le plan pragmatique, et sur le plan syntaxique il est seul sous la rection du verbe croire. En (17), une étudiante rapporte le discours "paniqué » de son camarade tricheur lors d'une épreuve écrite, pour laquelle il avait préparé des anti-sèches qu'il ne retrouvait plus dans ses vêtements :

(17) donc il fait comme ça $=-$ je sais que hein $\backslash /$ - je sais que la partie-là c'est où $\Lambda$ donc tu [rire] il copie les cours par chapitres $\backslash$ - tu vois $\backslash /$ - donc il a les $\backslash$ - je sais que / - quoi $\backslash$ - je sais que: / - waab $=-c^{\prime} e s t$ c'est $x x \backslash$ - je dis merde yakan tu es fort $\Lambda$ - quand il te tire l'habit / - il peut déchirer ça $\backslash$ (R3.AYALM)

Les énoncés en italiques correspondent à des que-I de type II. Certains sont réduits à un seul terme (je sais que hein, je sais que: - waah - c'est c'est xx). Si nous procédons à une mise en grille des énoncés, nous obtenons ce qui suit :

\begin{tabular}{|c|c|c|}
\hline (2) & $\begin{array}{r}\text { tu crois que } \\
\text { qu' }\end{array}$ & $\begin{array}{l}\text { quoi } \backslash \\
\text { on est vos égaux } \backslash\end{array}$ \\
\hline (16) & $\begin{array}{l}\text { je sais que heir } \\
\text { je sais que } \\
\text { je sais que/ - } \\
\text { je sais que:/ - }\end{array}$ & $\begin{array}{l}\text { la partie-là c'est où } / \\
\text { quoi } \backslash- \\
\text { waah }=- \text { c'est c'est xx } \backslash\end{array}$ \\
\hline
\end{tabular}

La prosodie nous aide pour le dégroupement, et (même de manière perceptive, sans analyse acoustique), les pauses entre que et quoi dans certains de ces énoncés montrent que les deux éléments peuvent être répartis en deux groupes intonatifs distincts. D'un point de vue macro-syntaxique, nous pouvons voir là une configuration de type noyau + suffixe, où le premier terme délimité par que constitue le noyau, et le deuxième le suffixe. Ainsi analysé, l'autonomie du noyau de certaines unités macrosyntaxiques semble difficile à établir. Mais, dans un contexte interactionnel comme en (16), la situation peut favoriser ou non l'autonomie du noyau. Ainsi, un noyau tel que $j e$ sais que: / suivi du postfixe waah -, est parfaitement valable.

Que sous la rection du verbe (savoir, croire, dire...) annonce un commentaire (c'est la fonction du suffixe) sur ce qui est cru, dit, pensé, su, commentaire apporté par quoi ou toute autre forme proportionnelle.

\subsection{Les formes de type III}

Comme pour les autres types, les tests de rection sont inopérants.

(3a) on bagarre que j'ai la force pour bagarrer $\backslash /$

(3b) ? c'est on bagarre que j'ai la force de bagarrer?

? on bagarre que ça? / on bagarre ça? 
Sur le plan discursif, on bagarre reprend un noyau précédemment énoncé par le locuteur L2 : je suis venu là pour qu'on bagarre $\wedge$. Cette reprise vise non pas à expliquer la première énonciation comme dans les cas précédents, mais à la commenter en quelque sorte, commentaire fourni par le suffixe j'ai la force de bagarrer. On a donc une configuration noyau + suffixe :

\begin{tabular}{|c|c|}
\hline noyau & suffixe \\
\hline On bagarre que & J'ai la force de bagarrer \\
\hline
\end{tabular}

Le place de que au sein du noyau est justifiée encore une fois par des paramètres d'ordre intonatif, entre autres. Il est notamment possible d'avoir une pause entre que et le suffixe, ou un marqueur discursif particulier.

\section{(3c) on bagarre que mon frère $\triangle$-j'ai la force de bagarrer $\backslash /$}

Que introduit un commentaire que l'énonciation du noyau appelle. Sur le plan sémantique, il participe, avec l'intonation finale, à la modalité interrogative de l'énoncé. Sur le plan pragmatique, il engage non une demande d'information, mais une modalité polémique de la question, car il instaure une « contestation » quant à la valeur de vérité d'une précédente énonciation.

Dans certaines conditions de discours, l'articulation segmentale du noyau peut «avorter»; le noyau est alors réduit au seul que. Si l'énoncé conserve toute sa force illocutoire, c'est que le rôle de que est bien de reprendre un noyau antérieur, tout en le modulant.

\section{Conclusion}

Notre description syntaxique de certaines interrogatives en français parlé au Cameroun constitue plus un programme qu'une démarche aboutie. L'objectif était simplement de rendre compte, à partir d'une perspective différente, des phénomènes de langue à propos desquels l'analyse grammaticale classique s'avère inopérante, compte tenu de la spécificité des données. Les réponses aux questions soulevées par la « complexité » des données ne manquent pas d'intérêt. Avec la macro-syntaxe, et sans disqualifier la syntaxe de rection, on rend possible de décrire la forme des énoncés d'un point de vue syntaxique sur un domaine plus vaste que celui de la rection. La recherche sur les français périphériques, ceux d'Afrique en particulier, peut trouver là un outil fécond de description pour l'étude de la variation du français. 


\section{RÉFÉRENCES BIBLIOGRAPHIQUES}

Avanzi, M. (2007), « Regards croisés sur la notion de macro-syntaxe », TraNeL 47, 39-58.

Blanche-Benveniste, C., Bilger M., Rouget C. et VAn Den Eynde K., (1990), Le Français parlé: Etudes grammaticales, Paris, Editions du C.N.R.S., coll. Sciences du Langage.

Deulofeu, J. (1999), «Questions de méthode dans la description morphosyntaxique de l'élément que en français contemporain ", Recherches sur le français parlé 15, 163-198.

Ducrot, O., (1984), Le dire et le dit. Paris : Editions de minuit.

GADET, F. (1997), Le français populaire, Paris : PUF.

ITALIA, M. (2005), «Variations en fonction de l'âge et du degré de scolarisation dans le français oral des locuteurs gabonais : la question du discours rapporté », in Ploog K. et Rui B., Appropriations du français en contexte multilingue, Éléments pour une réflexion didactique à propos des situations africaines, Besançon, P.U. Franche-Comté, 191-207.

Kerbrat-Orecchioni, K (dir), (1991), La question. Lyon: PUL. coll. Linguistique et sémiotique.

LE GOFFIC, P. (1992), "Que en français : essai de vue d'ensemble », Travaux de linguistique du CERLICO 5, 43-71.

Morel, M.-A. et Danon-Boileau, L. (1998), Grammaire de l'intonation, l'exemple du français, Gap, Ophrys.

NGamountsiKa, E. (2007), «Un aspect morphosyntaxique du français parlé au Congo : la question du discours rapporté », Revue de l'Université de Moncton Numéro hors série, 169-187.

Ploog, K. (2004), «Stratégies et structures du discours rapporté dans les récits abidjanais », in Lopez Muñoz, J. M., Marnette, S. \& Rosier, L., (éds), le Discours rapporté dans tous ses états, Paris, L'Harmattan, 297-306.

QUEFFÉLEC, A. (2006), « Restructurations morphosyntaxiques en français populaire camerounais : l'expression des modalités injonctives et interrogatives dans le discours rapporté », Le Français en Afrique 21, 267-280.

SouOP, A. (2002), L'interrogation en français camerounais, Mémoire de DEA. Université Stendhal de Grenoble.

\section{Annexe : Conventions de transcription}

Pause courte, moyenne ou longue --- ---

Allongement d'une voyelle :

Syllabe inaudible $\mathrm{x}$

Séquence de deux syllabes inaudibles $\mathrm{xx}$

Séquence de plusieurs syllabes inaudibles xxx

Les syllabes avortées sont signalées par une apostrophe '

Intonation basse en fin d'énoncé

Intonation modulée bas-haut en fin d'énoncé $\backslash /$

Intonation plate en fin d'énoncé $=$

Intonation haute en fin d'énoncé /

Intonation modulée haut-bas en fin d'énoncé $\bigwedge$ 